TRANSACTIONS OF THE

AMERICAN MATHEMATICAL SOCIETY

Volume 351, Number 2, February 1999, Pages 497-513

S $0002-9947(99) 01862-0$

\title{
ON SOME BANACH SPACE PROPERTIES SUFFICIENT FOR WEAK NORMAL STRUCTURE AND THEIR PERMANENCE PROPERTIES
}

\author{
BRAILEY SIMS AND MICHAEL A. SMYTH
}

\begin{abstract}
We consider Banach space properties that lie between conditions introduced by Bynum and Landes. These properties depend on the metric behavior of weakly convergent sequences. We also investigate the permanence properties of these conditions.
\end{abstract}

\section{INTRODUCTION}

Throughout this paper, $X$ will denote a real Banach space.

Recall that $X$ has (weak) normal structure if whenever $C$ is a (weak compact) bounded convex subset of $X$ with $\operatorname{diam} C>0$ then $\operatorname{rad} C<\operatorname{diam} C$, where

$$
\operatorname{diam} C:=\sup \{\|x-y\|: x, y \in C\} \quad \text { and } \quad \operatorname{rad} C:=\inf _{x \in C} \sup \{\|x-y\|: y \in C\}
$$

are the diameter and radius of the set $C$. Recall also that Banach spaces with the Schur property are those for which weak compact and norm compact sets coincide. This gives the well known fact that Schur spaces have weak normal structure. We will assume in the sequel that the Banach spaces $X$ are not Schur. Thus, they have weakly convergent sequences that are not norm convergent. This will also be convenient for the definition of some Banach space constants.

It is well known that $X$ fails weak normal structure if and only if there exists a sequence $\left(x_{n}\right)$ in $X$ with $x_{n} \stackrel{w}{\longrightarrow} 0$, diam $\overline{\operatorname{co}}\left\{x_{n}\right\}_{n=1}^{\infty}\left(=\operatorname{diam}\left\{x_{n}\right\}_{n=1}^{\infty}\right)=1$ and $\operatorname{dist}\left(x_{n+1}, \overline{\mathrm{co}}\left\{x_{k}\right\}_{k=1}^{n}\right) \rightarrow 1$.

In particular $\operatorname{diam}_{a}\left(x_{n}\right), \operatorname{rad}_{a}\left(x_{n}\right)$ and $\lim _{n}\left\|x_{n}\right\|$ are all equal to 1 , where

$$
\operatorname{diam}_{a}\left(x_{n}\right):=\lim _{n} \operatorname{diam}\left\{x_{k}\right\}_{k=n}^{\infty}
$$

and

$$
\operatorname{rad}_{a}\left(x_{n}\right):=\inf \left\{\limsup \left\|x-x_{n}\right\|: x \in \overline{\operatorname{co}}\left\{x_{n}\right\}_{n=1}^{\infty}\right\}
$$

are, respectively, the asymptotic diameter of $\left(x_{n}\right)$ and the asymptotic radius of $\left(x_{n}\right)$ in $\overline{\mathrm{co}}\left\{x_{n}\right\}_{n=1}^{\infty}$.

See [10] for details and the relevance of weak normal structure to fixed point theory of nonexpansive mappings.

In section 2 we discuss the notion of minimal sequential diameter, which will be useful as a technique in the investigation of metric phenomena in the sequel and is also used explicitly in section 3 for the definition of Banach space properties.

Received by the editors November 27, 1995 .

1991 Mathematics Subject Classification. Primary 47H09, 47H10, 46B20.

(C)1999 American Mathematical Society 
Section 3 considers the properties mentioned in the abstract. Section 4 is devoted to permanence results for these properties.

\section{ON THE Minimal SEQUENTIAL DiAMETER}

Throughout this section $C$ will denote a nonempty bounded metric space. Define, for infinite such $C$,

$$
\begin{aligned}
A^{*}(C) & :=\inf \{\operatorname{diam} B: B \text { is an infinite subset of } C\} \\
& =\inf \left\{\operatorname{diam}\left(x_{n}\right):\left(x_{n}\right) \text { is an infinite sequence in } C\right\},
\end{aligned}
$$

the equality being obvious. For convenience, if $C$ is finite, put $A^{*}(C)=0$. We will call $A^{*}(C)$ the minimal sequential diameter of $C$.

The minimal sequential diameter will be related in this section to the separation index of noncompactness, and also to the notion of $\alpha$-minimal sets. It is also used in section 3 for the definition of Banach space properties.

We recall the following combinatorial result.

Ramsey's Theorem 2.1. Suppose $n \in \mathbb{N}$ and $D$ is an infinite set. With $\mathcal{P}_{n}(D)$ denoting the members of $\mathcal{P}(D)$ with $n$ elements, suppose that $\mathcal{P}_{n}(D)=A \cup B$. Then there exists an infinite subset $E$ of $D$ so that either $\mathcal{P}_{n}(E) \subseteq A$ or $\mathcal{P}_{n}(E) \subseteq B$ (the possibilities are not mutually exclusive).

See page 234 of [7] or 392 of [2] for a proof of this result.

Recall that the separation index of a bounded nonempty metric space $C$ is defined by

$$
\gamma(C):=\sup \left\{\operatorname{sep}\left(y_{n}\right):\left(y_{n}\right) \text { is a sequence in } C\right\},
$$

where $\operatorname{sep}\left(x_{n}\right):=\inf _{n \neq m} d\left(x_{n}, x_{m}\right)$ is the separation of a bounded sequence $\left(x_{n}\right)$.

Lemma 2.2. Suppose $C$ is infinite and $r \geq 0$. Then there exists an infinite sequence $\left(y_{n}\right)$ in $C$ so that either $\operatorname{diam}\left(y_{n}\right) \leq r$ or $\operatorname{sep}\left(y_{n}\right) \geq r$.

Proof. Let

$$
\begin{aligned}
& A=\left\{\{x, y\} \in \mathcal{P}_{2}(C): d(x, y) \leq r\right\}, \\
& B=\left\{\{x, y\} \in \mathcal{P}_{2}(C): d(x, y) \geq r\right\} .
\end{aligned}
$$

Using 2.1 when $n=2$ then gives the result.

We can now relate the minimal sequential diameter with the separation index of noncompactness.

Lemma 2.3. $A^{*}(C) \leq \gamma(C)$.

Proof. The lemma is trivial if $C$ is finite, so suppose that $C$ is infinite. Suppose now on the contrary that $\gamma(C)<r<A^{*}(C)$ for some $r>0$. But by using lemma 2.2 we can obtain an infinite sequence in $C$ whose diameter is no larger than $r$ or whose separation is no smaller than $r$. Either possibility will give a contradiction.

Proposition 2.4. If $C$ is an infinite bounded metric space, then

$$
\gamma(C)=\sup \left\{A^{*}(D): D \text { is an infinite subset of } C\right\}
$$

and

$$
A^{*}(C)=\inf \{\gamma(D): D \text { is an infinite subset of } C\} \text {. }
$$


Proof. From lemma 2.3, if $D$ is an infinite subset of $C$ then

$$
A^{*}(D) \leq \gamma(D) \leq \gamma(C) .
$$

Thus $\gamma(C) \geq \sup \left\{A^{*}(D): D\right.$ is an infinite subset of $\left.C\right\}$. To establish the reverse inequality, suppose that $\epsilon>0$. Then let $\left(x_{n}\right)$ be a sequence in $C$ with $\operatorname{sep}\left(x_{n}\right) \geq$ $\gamma(C)-\epsilon$. Clearly then $A^{*}\left(x_{n}\right) \geq \gamma(C)-\epsilon$, and the first equality is shown. Also we have, for any infinite subset $D$ of $C$,

$$
A^{*}(C) \leq A^{*}(D) \leq \gamma(D)
$$

and so $A^{*}(C) \leq \inf \{\gamma(D): D$ is an infinite subset of $C\}$. Since $\gamma(D) \leq \operatorname{diam} D$ for any subset $D$ of $C$, the reverse inequality is clear.

In [4] the notion of $\alpha$-minimality is defined. First recall that the $\alpha$ index of noncompactness of a nonempty bounded metric space $C$ is defined by

$\alpha(C):=\inf \{d: C$ can be covered with a finite number of sets of diameter $\leq d\}$.

An infinite bounded metric space $C$ is called $\alpha$-minimal if $\alpha(B)=\alpha(C)$ for every infinite subset $B$ of $C$. It is shown in [4] that every infinite bounded metric space contains an $\alpha$-minimal subset. We now verify this using an alternative method.

After [22] we call a bounded sequence $\left(x_{n}\right)$ in $C$ asymptotically equidistant if

$$
\lim _{n, m \rightarrow \infty(n \neq m)} d\left(x_{n}, x_{m}\right) \quad \text { exists. }
$$

The following lemma is widely known and will be useful in the sequel. The proof we give is suggested in [5].

Lemma 2.5. Every bounded infinite metric space $C$ contains an asymptotically equidistant sequence.

Proof. Let $\epsilon_{n}>0, \epsilon_{n} \rightarrow 0$. Choose an infinite sequence $\left(x_{n}\right)$ from $C$ so that $\operatorname{sep}\left(x_{n}\right) \geq \gamma(C)-\epsilon_{1} / 2$. Lemma 2.2 will give a subsequence $\left(y_{n}\right)$ of $\left(x_{n}\right)$ such that $\operatorname{diam}\left(y_{n}\right) \leq \gamma(C)+\epsilon_{1} / 2$.

Thus $\gamma(C)-\epsilon_{1} / 2 \leq d\left(y_{n}, y_{m}\right) \leq \gamma(C)+\epsilon_{1} / 2$ for $n \neq m$.

We now continue the process, using $\left(y_{n}\right)$ and $\epsilon_{2}$ instead of $C$ and $\epsilon_{1}$, etc. A diagonalization will then give the result.

Proposition 2.6. Every infinite bounded metric space contains an $\alpha$-minimal subset.

Proof. By the previous lemma it is sufficient to show that an asymptotically equidistant sequence is $\alpha$-minimal. So, suppose that $\lim _{n, m \rightarrow \infty(n \neq m)} d\left(x_{n}, x_{m}\right)=l$. Since any subsequence of $\left(x_{n}\right)$ also has this property, it is sufficient to show that $\alpha\left(x_{n}\right)=l$. But this is easily seen to be so.

The next proposition shows that $\alpha$-minimal sets can be easily characterized using the minimal sequential diameter. Its truth is obvious.

Proposition 2.7. If $C$ is infinite then

$$
A^{*}(C)=\inf \{\alpha(B): B \text { is an infinite subset of } C\} .
$$

Also, $C$ is $\alpha$-minimal if and only if

$$
A^{*}(C)=\alpha(C)
$$


Note that lemma 2.3 and proposition 2.7 together imply that for an $\alpha$-minimal set $C, A^{*}(C)=\gamma(C)=\alpha(C)$. Simple examples show that the last equality does not characterize $\alpha$-minimal sets.

We now recover lemma 1 of [23].

Proposition 2.8. If $C$ is infinite, then

$$
\gamma(C)=\sup \{\alpha(B): B \text { is an } \alpha \text {-minimal subset of } C\} .
$$

$\alpha(B)$ can be replaced by $A^{*}(B)$ or $\gamma(B)$ in the above equality.

Proof. We have from 2.4 that

$$
\gamma(C)=\sup \left\{A^{*}(D): D \text { is an infinite subset of } C\right\} .
$$

Write $s=\sup \{\alpha(B): B$ is a $\alpha$-minimal subset of $C\}$. By proposition 2.6 an infinite subset $D$ of $C$ contains an $\alpha$-minimal subset $B .2 .7$ then gives that

$$
A^{*}(D) \leq A^{*}(B)=\alpha(B) .
$$

Thus $s \geq \gamma(C)$. The reverse inequality is also given by proposition 2.7. The remainder of the proposition is now established by using the remark preceding it.

\section{Some Banach SPACE Properties}

Bynum [6] defined the weakly convergent sequence coefficient of a Banach space $X$ to be

$$
W C S(X):=\inf \left\{\frac{\operatorname{diam}_{a}\left(x_{n}\right)}{\operatorname{rad}_{a}\left(x_{n}\right)}: x_{n} \stackrel{w}{\longrightarrow} 0, x_{n} \nrightarrow 0\right\} .
$$

It is easily checked that $\operatorname{diam}_{a}$ can be replaced with diam in the definition.

Some authors have said that a space $X$ has weak uniform normal structure if $W C S(X)>1$. We shall say that $X$ satisfies Bynum's condition if this inequality holds.

We need some further notation for the following proposition. With $D\left[\left(x_{n}\right)\right]:=$ $\lim \sup _{n} \lim \sup _{m}\left\|x_{n}-x_{m}\right\|$ for a bounded sequence $\left(x_{n}\right)$, [12] defines

$$
\beta(X):=\inf \left\{D\left[\left(x_{n}\right)\right]:\left\|x_{n}\right\| \rightarrow 1, x_{n} \stackrel{w}{\longrightarrow} 0\right\} .
$$

Most of the contents of the following proposition has been noticed before.

Proposition 3.1. The following are equal.

(1) $W C S(X)$.

(2) $\inf \left\{\operatorname{diam}_{a}\left(x_{n}\right): x_{n} \stackrel{w}{\longrightarrow} 0,\left\|x_{n}\right\| \rightarrow 1\right\}$.

(3) $\beta(X)$.

(4) $\inf \left\{\gamma\left(x_{n}\right): x_{n} \stackrel{w}{\longrightarrow} 0,\left\|x_{n}\right\| \rightarrow 1\right\}$.

(5) $\inf \left\{\alpha\left(x_{n}\right): x_{n} \stackrel{w}{\longrightarrow} 0,\left\|x_{n}\right\| \rightarrow 1\right\}$.

(6) $\inf \left\{A^{*}\left(x_{n}\right): x_{n} \stackrel{w}{\longrightarrow} 0,\left\|x_{n}\right\| \rightarrow 1\right\}$.

(7) $\inf \left\{\lim _{n, m \rightarrow \infty, n \neq m}\left\|x_{n}-x_{m}\right\|: x_{n} \stackrel{w}{\longrightarrow} 0,\left\|x_{n}\right\| \rightarrow 1\right.$ and $\lim \left\|x_{n}-x_{m}\right\|$ exists\}.

Proof. That $(1)=(2)$ is well known (see, for example, [18],[22]). Lemma 2.5 gives $(4)=(7)$. Obviously $(2)=(5)=(6)$ on the extraction of appropriate subsequences. Also $(6) \leq(4)$ by 2.4 . Finally, $(4) \leq(3) \leq(2)$ is straightforward and completes the proof. 
Note. The condition that $\left\|x_{n}\right\| \rightarrow 1$ in the above can be replaced by the condition that $\left\|x_{n}\right\|=1$ for all $n$.

We should note that the equality of (1) with (4) was noted in [18], and that of (1) with (7) in [5] and [22].

A large class of Banach spaces have Bynum's condition. Spaces which have uniform normal structure have this condition. A Banach space $X$ has uniform normal structure if

$$
\inf \left\{\frac{\operatorname{diam} C}{\operatorname{rad} C}: C \text { is a bounded convex subset with } \operatorname{diam} C>0\right\}>1 .
$$

Uniformly convex spaces have uniform normal structure and thus Bynum's condition. We mention now some properties weaker than uniform convexity.

$X$ is Nearly Uniformly Convex (NUC) if, given $\epsilon>0$, there exists $\delta(\epsilon)>0$ so that if $\left(x_{n}\right)$ is a sequence in $B_{X}$ and $\operatorname{sep}\left(x_{n}\right)>\epsilon$, then $\overline{\operatorname{co}}\left(x_{n}\right) \cap B_{1-\delta}(0) \neq \emptyset$.

It will be convenient to introduce the following modulus. If $\epsilon>0$ let

$$
\begin{array}{r}
\diamond(\epsilon):=\inf \left\{1-\|x\|: \text { there exists a sequence }\left(x_{n}\right) \subseteq B_{X}\right. \\
\text { with } \left.x_{n} \stackrel{w}{\longrightarrow} x \text { and } \operatorname{sep}\left(x_{n}\right)>\epsilon\right\} .
\end{array}
$$

It is implicit that we only allow $\epsilon$ 's for which the set used in the definition is nonempty. We say that $X$ is Uniformly Kadec-Klee (UKK) if $\diamond(\epsilon)>0$ for all $\epsilon$, $\epsilon$-UKK if $\diamond(\epsilon)>0$ and $\epsilon^{-}$-UKK if $\lim _{h \rightarrow \epsilon^{-}} \diamond(h)>0$. Of course $X$ is $\epsilon^{-}-\mathrm{UKK}$ exactly when there exists a $h<\epsilon$ so that $\diamond(h)>0$.

It is shown in [11] that $X$ is NUC if and only if $X$ is UKK and reflexive, and that in general $\mathrm{UC} \Rightarrow \mathrm{NUC} \Rightarrow \mathrm{UKK}$, with none of these implications reversible.

In [8] van Dulst and Sims called a Banach space $X$ Weakly Uniformly KadecKlee (WUKK) if there exist an $\epsilon<1$ and a $\delta>0$ so that if $\left(x_{n}\right) \subseteq B_{X}$ and $x_{n} \stackrel{w}{\longrightarrow} x$ with $\operatorname{sep}\left(x_{n}\right)>\epsilon$ then $\|x\| \leq 1-\delta$. By the above, this is equivalent to $X$ being $1^{-}-\mathrm{UKK}$.

Landes [14] defined WUKK', which results from replacing $\operatorname{sep}\left(x_{n}\right)>\epsilon$ in the definition of WUKK by $\liminf \left\|x_{n}-x\right\|>\epsilon$. On the extraction of appropriate subsequences it can be seen that lim inf can be replaced by lim sup in the definition and WUKK' can be written as: There exist an $\epsilon<1$ and a $\delta>0$ so that if $x_{n} \in B_{X}$ and $x_{n} \stackrel{w}{\longrightarrow} x$, then $\|x\| \leq 1-\delta$ if limsup $\left\|x_{n}-x\right\|>\epsilon$.

That WUKK implies WUKK' is clear, since any sequence $\left(x_{n}\right)$ converging weakly to $x$ satisfies $\gamma\left(x_{n}\right) \geq \liminf \left\|x_{n}-x\right\|$ (using the weak lower semicontinuity of the norm).

Proposition 3.2. $W U K K^{\prime}$ is equivalent to each of the following.

(1) There exist $\epsilon<1, \delta>0$ so that if limsup $\left\|x_{n}-x\right\| \leq 1, x_{n} \stackrel{w}{\longrightarrow} 0$ and limsup $\left\|x_{n}\right\|>\epsilon$, then $\|x\| \leq 1-\delta$.

(2) There exist $r<1, \rho>1$ so that if $\left\|x_{n}\right\| \rightarrow 1, x_{n} \stackrel{w}{\longrightarrow} 0$ and $\|x\|>r$, then limsup $\left\|x_{n}-x\right\| \geq \rho$.

(3) There exist $r<1, \rho>1$ so that if $\lim \sup \left\|x_{n}\right\| \geq 1, x_{n} \stackrel{w}{\longrightarrow} 0$ and $\|x\|>r$, then limsup $\left\|x_{n}-x\right\| \geq \rho$.

Proof. WUKK $\Rightarrow(1)$ : Suppose $X$ is WUKK'. Let $\delta$ and $\epsilon$ be the associated constants. Now suppose that limsup $\left\|x_{n}-x\right\| \leq 1, x_{n} \stackrel{w}{\longrightarrow} 0$ and lim $\sup \left\|x_{n}\right\|>\epsilon$. We show that $\|x\| \leq 1-\delta$. 
Clearly we can assume that $\left\|x_{n}-x\right\| \rightarrow s \leq 1$ and that $s>0,\left\|x_{n}-x\right\|>0$ for all $n$. Now, with

$$
y_{n}:=\frac{x_{n}-x}{\left\|x_{n}-x\right\|},
$$

we have $\left\|y_{n}\right\|=1, y_{n} \stackrel{w}{\longrightarrow}-x / s$ and $\lim \sup \left\|y_{n}+x / s\right\|>\epsilon / s \geq \epsilon$.

Thus by WUKK ${ }^{\prime},\|x / s\| \leq 1-\delta$. Thus $\|x\| \leq(1-\delta) s \leq 1-\delta$ as required.

(1) $\Rightarrow$ WUKK': Suppose that $X$ satisfies condition (1) and that $\left\|y_{n}\right\| \leq 1$, $y_{n} \stackrel{w}{\longrightarrow} y$ with limsup $\left\|y_{n}-y\right\|>\epsilon$.

Then, putting $x_{n}:=y_{n}-y, x:=-y$, we have $x_{n} \stackrel{w}{\longrightarrow} 0$, limsup $\left\|x_{n}\right\|>\epsilon$ and $\lim \sup \left\|x_{n}-x\right\|=\lim \sup \left\|y_{n}\right\| \leq 1$. Thus $\|x\| \leq 1-\delta$, giving $\|y\| \leq 1-\delta$ and WUKK'.

$(1) \Rightarrow(2)$ : Suppose that $X$ has condition (1) with the associated $\epsilon$ and $\delta$. We can assume that $1-\delta<\epsilon$.

Now suppose that $\|x\|>(1-\delta) / \epsilon,\left\|x_{n}\right\| \rightarrow 1$ and $x_{n} \stackrel{w}{\longrightarrow} 0$.

Then suppose that $\lim \sup \left\|x_{n}-x\right\|<1 / \epsilon, s>1$ with $\lim \sup \left\|s \epsilon x_{n}-s \epsilon x\right\|<1$. Now $s \epsilon x_{n} \stackrel{w}{\longrightarrow} 0$ and limsup $\left\|s \epsilon x_{n}\right\|=s \epsilon>\epsilon$. Thus (1) gives $\|s \epsilon x\| \leq 1-\delta$, i.e. $\|x\| \leq(1-\delta) / s \epsilon<(1-\delta) / \epsilon$, a contradiction.

Thus limsup $\left\|x_{n}-x\right\| \geq 1 / \epsilon$, giving (2) with $r=(1-\delta) / \epsilon$ and $\rho=1 / \epsilon$.

$(2) \Rightarrow(3)$ : Suppose that (2) holds with $r$ and $\rho$ given. Now suppose that $\lim \sup \left\|x_{n}\right\| \geq 1, x_{n} \stackrel{w}{\longrightarrow} 0$ and $\|x\|>r$, but that limsup $\left\|x_{n}-x\right\|<\rho$.

Since $x_{n} \stackrel{w}{\longrightarrow} 0,\|x\|<\rho$. We can assume that $\left\|x_{n}\right\| \rightarrow a \geq 1$. Then

$$
\begin{aligned}
\limsup \left\|\frac{x_{n}}{a}-x\right\| & =\limsup \left\|\frac{1}{a}\left(x_{n}-x\right)+\left(1-\frac{1}{a}\right)(-x)\right\| \\
& <\frac{\rho}{a}+\left(1-\frac{1}{a}\right) \rho \\
& =\rho .
\end{aligned}
$$

But $x_{n} / a \stackrel{w}{\longrightarrow} 0$ and $\left\|x_{n} / a\right\| \rightarrow 1$, contradicting (2). Thus $X$ satisfies condition (3).

$(3) \Rightarrow(1)$ : Suppose $r$ and $\rho$ are given by (3). Assume that limsup $\left\|x_{n}-x\right\| \leq$ $1, x_{n} \stackrel{w}{\longrightarrow} 0$ and $\lim \sup \left\|x_{n}\right\|>1 / \rho$. Choose $t$ so that $\rho \lim \sup \left\|x_{n}\right\|>t>1$.

Then $\lim \sup \left\|\left(\rho x_{n}\right) / t\right\|>1$, limsup $\left\|\rho x_{n} / t-\rho x / t\right\| \leq \rho / t<\rho$ and $\rho x_{n} / t \stackrel{w}{\longrightarrow} 0$; so by (3), $\|\rho x / t\| \leq r$. Thus $\|x\| \leq t r / \rho$. Since $t$ can be made arbitrarily close to 1 , $\|x\| \leq r / \rho$. Thus $X$ has (1) with $\epsilon=1 / \rho$ and $\delta=1-r / \rho$.

Propositions 2.1 and 3.2 tell us that WUKK' implies Bynum's condition. We note that this was also essentially shown in [14].

We now define other conditions that depend on the metric properties of weak null sequences. Perhaps the most recently introduced property is property $(\mathrm{P})$ of Tan and $\mathrm{Xu}[20]$ :

$$
\lim \inf \left\|x_{n}-x\right\|<\operatorname{diam}\left(x_{n}\right) \quad \text { if } \quad x_{n} \stackrel{w}{\longrightarrow} x \quad \text { and } \quad\left(x_{n}\right) \quad \text { is nonconstant. }
$$

By extracting appropriate subsequences this can be seen to be unaltered if lim sup is used instead of liminf and, on normalizing, is equivalent to:

$$
\text { If }\left\|x_{n}\right\| \rightarrow 1 \quad \text { and } \quad x_{n} \stackrel{w}{\longrightarrow} 0 \text { then } \operatorname{diam}\left(x_{n}\right)>1 .
$$


We say that $X$ has asymptotic $(\mathrm{P})$ if the above (again equivalent) conditions hold with diam replaced by $\operatorname{diam}_{a}$ (assuming that $\left(x_{n}\right)$ is nonconvergent). Clearly asymptotic $(\mathrm{P}) \Rightarrow(\mathrm{P})$, and asymptotic $(\mathrm{P})$ can also be written as:

$$
\text { If }\left\|x_{n}\right\|=1 \quad \text { and } \quad x_{n} \stackrel{w}{\longrightarrow} 0, \text { then } \operatorname{diam}_{a}\left(x_{n}\right)>1 .
$$

In [19] it was noted that asymptotic $(\mathrm{P})$ and $(\mathrm{P})$ are distinct properties, and that $(\mathrm{P})$ is equivalent to a condition introduced in [21] which has subsequently been known as WO (since it is a weakening of the Opial condition [17]): $X$ has WO if, given a nonconstant weak null sequence, $\lim \inf \left\|x_{n}\right\|<\sup _{m} \lim \sup _{n}\left\|x_{m}-x_{n}\right\|$.

WO can be restated as follows: If $x_{n} \stackrel{w}{\longrightarrow} 0$ and $\left(x_{n}\right)$ is a nonconstant sequence, then there exists $x \in \overline{\mathrm{co}}\left(x_{n}\right)$ so that $\lim \sup \left\|x_{n}\right\|<\lim \sup \left\|x-x_{n}\right\|$. Of course WO can also be normalized as $(\mathrm{P})$ was.

In [12] a Banach space was said to satisfy the Generalized Gossez-Lami Dozo property (GGLD) if the original inequality defining WO holds with $\sup _{m}$ replaced by $\lim \sup _{m}$. The following was proved in [19].

Proposition 3.3. The following statements are equivalent.

(1) $X$ has asymptotic $(P)$.

(2) $X$ has the $G G L D$ condition.

(3) If $x_{n} \in X$ and $x_{n} \stackrel{w}{\longrightarrow} 0,\left\|x_{n}\right\| \rightarrow 1$, then $\gamma\left(x_{n}\right)>1$.

(4) If $x_{n} \in X$ and $x_{n} \stackrel{w}{\longrightarrow} 0,\left\|x_{n}\right\| \rightarrow 1$, then $\alpha\left(x_{n}\right)>1$.

We will say that $X$ has Subsequential (P) (SuP) if, when $x_{n} \stackrel{w}{\longrightarrow} 0$ and $\left\|x_{n}\right\| \rightarrow 1$, that $A^{*}\left(x_{n}\right)>1$.

We also say that $X$ has Strong Subsequential (P) (SSuP) if and only if, given a weak null sequence $\left(x_{n}\right)$ in $X$ with $\lim \inf \left\|x_{n}\right\|>0$, there exists an $r<1$ so that if $\left(y_{n}\right)$ is any subsequence of $\left(x_{n}\right)$, then $\lim \sup \left\|y_{n}\right\| \leq r \operatorname{diam}\left(y_{n}\right)$.

$\mathrm{SSuP}$ is perhaps a more natural property than SuP. It is shown in section 4 that it is preserved under finite products. Clearly Bynum's condition $\Rightarrow \mathrm{SSuP} \Rightarrow \mathrm{SuP}$ $\Rightarrow$ asymptotic $(\mathrm{P})$. It is also shown in section 4 that the first two properties, as well as the last two, are distinct. It is unknown whether SSuP is different from SuP. We now give an elementary restatement of SSuP that will be useful later.

Proposition 3.4. SSuP is equivalent to:

If $x_{n} \stackrel{w}{\longrightarrow} 0$ and $\liminf \left\|x_{n}\right\|>0$, then there exists $s>0$ so that $\operatorname{diam}\left(y_{n}\right) \geq$ limsup $\left\|y_{n}\right\|+s$ for every subsequence $\left(y_{n}\right)$ of $\left(x_{n}\right)$.

Proof. Suppose that $X$ has SSuP and $x_{n} \stackrel{w}{\longrightarrow} 0$, liminf $\left\|x_{n}\right\|>0$. Then there exists $r<1$ so that $\lim \sup \left\|y_{n}\right\| \leq r \operatorname{diam}\left(y_{n}\right)$ for every subsequence $\left(y_{n}\right)$ of $\left(x_{n}\right)$. For such $\left(y_{n}\right)$, then,

$$
\begin{aligned}
\operatorname{diam}\left(y_{n}\right) & \geq \frac{\limsup \left\|y_{n}\right\|}{r} \\
& =\limsup \left\|y_{n}\right\|+\lim \sup \left\|y_{n}\right\|\left(\frac{1}{r}-1\right) \\
& \geq \limsup \left\|y_{n}\right\|+\liminf \left\|x_{n}\right\|\left(\frac{1}{r}-1\right) .
\end{aligned}
$$

Thus liminf $\left\|x_{n}\right\|((1 / r)-1)$ suffices for $s$.

Conversely, suppose that $X$ satisfies the statement in the proposition and $x_{n} \stackrel{w}{\longrightarrow}$ $0, \liminf \left\|x_{n}\right\|>0$. Let $s$ be as given by the statement. Then for any subsequence 
$\left(y_{n}\right)$ of $\left(x_{n}\right)$ we have $\limsup \left\|y_{n}\right\| \leq \operatorname{diam}\left(y_{n}\right)-s$. Now, since $\left(x_{n}\right)$ is bounded, there exists $r<1$ so that

$$
\frac{\operatorname{diam}\left(y_{n}\right)-s}{\operatorname{diam}\left(y_{n}\right)} \leq r
$$

for any subsequence $\left(y_{n}\right)$ of $\left(x_{n}\right)$. Then $\lim \sup \left\|y_{n}\right\| \leq r \operatorname{diam}\left(y_{n}\right)$, as required.

We now show that Bynum's condition, SSuP and SuP are equivalent in the class of Asplund spaces.

Theorem 3.5. If $X$ is an Asplund space and $W C S(X)=1$, then $X$ does not have SuP.

Proof. We will use the characterization of an Asplund space as one in which separable subspaces have separable duals. Suppose then that $X$ is Asplund and $W C S(X)$ $=1$. Then we can obtain a sequence $\left(x^{m}\right)$ of weak null sequences $x^{m}=\left(x_{n}^{m}\right)$ satisfying $\lim _{n}\left\|x_{n}^{m}\right\|=1$ and $\operatorname{diam}\left(x_{n}^{m}\right)_{n=1}^{\infty} \leq 1+1 / m$ for all $m$. Since we are working in a separable subspace of $X$, we can assume that $X$ has separable dual. But then bounded subsets of $X$ are metrizable and thus first countable in the weak topology. We can clearly assume that $C:=\left\{x_{n}^{m}\right\}_{n, m \in \mathbb{N}}$ is bounded.

Let $\left\{A_{m}\right\}$ and $\left\{B_{m}\right\}$ be, respectively, nested bases for the weak topology (restricted to $C$ ) at 0 and the euclidean topology at 1 . Without loss of generality we can assume that $x_{n}^{m} \in A_{m}$ and $\left\|x_{n}^{m}\right\| \in B_{m}$ for any $n, m$. It now follows that any enumeration of $C$ gives a weak null sequence (w-ns) with norms converging to 1 and which violates $\mathrm{SuP}$.

In [21] it was asked whether $\mathrm{WO}$ is equivalent to $\mathrm{w}-\mathrm{ns}$. It is easily seen, given the equivalence of $(\mathrm{P})$ and $\mathrm{WO}$, that this is true in the class of spaces with the nonstrict Opial condition ( $X$ has the nonstrict Opial condition if $x_{n} \stackrel{w}{\longrightarrow} 0$ and $\left\|x_{n}\right\| \rightarrow 1$ imply limsup $\left\|x-x_{n}\right\| \geq 1$ for any $\left.x \in X\right)$.

We now comment on a property introduced by Landes that lies between $(\mathrm{P})$ and w-ns. Firstly, we establish some nomenclature. Suppose that $\left(x_{n}\right)$ is a bounded sequence. Then $\left(x_{n}\right)$ is said to be limit affine if $\lim \left\|x-x_{n}\right\|$ exists for all $x \in \overline{\mathrm{co}}\left(x_{n}\right)$ and the function $f: \overline{\mathrm{co}}\left(x_{n}\right) \rightarrow \mathbb{R}$ defined by $f(x)=\lim \left\|x-x_{n}\right\|$ is affine on $\overline{\mathrm{Co}}\left(x_{n}\right)$. The sequence is further said to be nondecreasingly limit affine if $\left(f\left(x_{n}\right)\right)$ is a nondecreasing sequence.

The Banach space $X$ is said to have the Weak Sum Property (WSP) if every weakly convergent nondecreasingly limit affine sequence in $X$ is constant. This is not the actual definition given in [16], but is equivalent to it by a theorem in [16].

Landes proved that WSP is the weakest Banach space property so that every finite product of spaces with this property has w-ns. In particular, he showed that WSP is preserved under the taking of finite products.

We note that $(\mathrm{P})$ implies WSP. Indeed, suppose that $\left(x_{n}\right)$ is a nonconstant weakly convergent (to 0 , without loss of generality) nondecreasingly limit affine sequence. Then $f\left(x_{n}\right)$ is convergent, to $l$ say. Since $f$ is affine and (norm) continuous on $\overline{\mathrm{CO}}\left(x_{n}\right)$, it is also weak continuous. Thus $f(0)=\lim \left\|x_{n}\right\|=l$. The fact that $f\left(x_{n}\right)$ is nondecreasing will now contradict ( $\left.\mathrm{P}\right)$ (or, more precisely, WO).

[16] also contains two examples that are of interest to us. They are, using Landes' notation for the norms, $\left(c_{0},\|\|_{1}\right)$ and $\left(c_{0},\|\|_{1,1}^{\prime}\right)$, both renormings of $c_{0}$, which we will denote by $X_{1}$ and $X_{2}$ respectively. It is shown in [16] that $X_{1}$ has WSP, and that $X_{2}$ has w-ns but not WSP. Also, it is easy to verify that the sequence of 
coordinate indicators $\left(e_{n}\right)$ in $X_{1}$ satisfies $\left\|e_{n}\right\|_{1} \rightarrow 1, e_{n} \stackrel{w}{\longrightarrow} 0$ and $\operatorname{diam}\left(e_{n}\right)=1$, giving that $X_{1}$ fails $(\mathrm{P})$. This shows that WSP lies strictly between $(\mathrm{P})$ and $\mathrm{w}-\mathrm{ns}$, and of course answers Tingley's question.

\section{Permanence properties}

Perhaps the first relevant product result was given in [3]: that the $\ell_{\infty}$ product of finitely many spaces with normal structure also has normal structure. Also Landes showed in [15] that normal structure is preserved when a general substitution space is uniformly convex. The methods of proof used for those results also give preservation of weak normal structure. We give results on the permanence properties of some of the conditions defined in previous sections. Included are infinite product results that are useful in separating some of the conditions considered in the previous section.

We first give finite product results. Suppose $Z$ is a finite dimensional normed space, written $Z=\left(\mathbb{R}^{m},\|\|_{Z}\right)$, which has a monotone norm. That is,

$$
\|(x(1), \ldots, x(m))\|_{Z} \leq\|(y(1), \ldots, y(m))\|_{Z} \text { if } 0 \leq x(i) \leq y(i) \text { for all } i .
$$

If $X_{1}, \ldots, X_{m}$ are $m$ Banach spaces, then the $Z$ direct product of them, written $\left(X_{1} \oplus \cdots \oplus X_{m}\right)_{Z}$, is the Banach space $\left(\prod_{i=1}^{m} X_{i},\|\|\right)$, where

$$
\|(x(1), \ldots, x(m))\|=\left\|\left(\|x(1)\|_{X_{1}}, \ldots,\|x(m)\|_{X_{m}}\right)\right\|_{Z}
$$

for $(x(1), \ldots, x(m)) \in \prod_{i=1}^{m} X_{i}$.

Proposition 4.1. Property $(P)$ is preserved under the taking of finite products.

Proof. Suppose $X_{1}, \ldots, X_{m}$ all have property $(\mathrm{P})$, and write $X=\left(X_{1} \oplus \cdots \oplus X_{m}\right)_{Z}$. Now suppose that $x_{n} \in X, x_{n} \stackrel{w}{\longrightarrow} 0$ and $\left\|x_{n}\right\| \rightarrow 1$. We will show that $\operatorname{diam}\left(x_{n}\right)>1$, giving $(\mathrm{P})$ for $X$.

Write $x_{n}=\left(x_{n}(1), \ldots, x_{n}(m)\right)$. Then $x_{n}(i) \stackrel{w}{\longrightarrow} 0$ for all $i$. We can assume that none of the $\left(x_{n}(i)\right)_{n=1}^{\infty}$ have any constant subsequences (otherwise $\left(x_{n}(i)\right)_{n=1}^{\infty}$ has a constantly 0 subsequence and we can disregard coordinate $i$ ).

We can also assume that $\left\|x_{n}(i)\right\| \rightarrow N(i)$, say, so that

$$
\|(N(1), \ldots, N(m))\|_{Z}=\lim _{n}\left\|\left(\left\|x_{n}(1)\right\|, \ldots,\left\|x_{n}(m)\right\|\right)\right\|_{Z}=\lim \left\|x_{n}\right\|=1 .
$$

Since the $X_{i}$ have property $(\mathrm{P})$, by 2.1 there exists a subsequence $\left(y_{n}\right)$ of $\left(x_{n}\right)$ so that $\left\|y_{n}(i)-y_{m}(i)\right\|>N(i)$ if $n \neq m$ and for any $i$ (noting that if $N(i)=0$ and property $(\mathrm{P})$ cannot be applied there is still no problem by the earlier assumption on the sequence $\left.\left(x_{n}\right)\right)$. This easily gives $\operatorname{diam}\left(y_{n}\right)>1$ and property $(\mathrm{P})$ for $X$.

Proposition 4.2. Asymptotic $(P)$ is preserved under the taking of finite products. Proof. We first follow the proof of 4.1 until just after the definition of $N(i)$. We show that $\gamma\left(x_{n}\right)>1$, thus establishing the result by 3.3 .

Now by 3.2 we can obtain a subsequence (calling it $\left(x_{n}\right)$ again) of $\left(x_{n}\right)$ so that $\operatorname{sep}\left(x_{n}(i)\right) \geq r N(i)$ for any $i$ and some $r>1$ (noting that this is satisfied automatically if $N(i)=0)$. Then, for any $p, n \in \mathbb{N}$,

$$
\begin{aligned}
\left\|x_{p}-x_{n}\right\| & =\left\|\left(\left\|x_{p}(1)-x_{n}(1)\right\|, \ldots,\left\|x_{p}(m)-x_{n}(m)\right\|\right)\right\|_{Z} \\
& \geq\|(r N(1), \ldots, r N(m))\|_{Z} \\
& >\|(N(1), \ldots, N(m))\|_{Z} \\
& =1
\end{aligned}
$$

completing the proof. 
Below we will find it convenient to use the following fact.

If for any $n \in \mathbb{N}$ we are given $x_{n}=\left(x_{n}(1), \ldots, x_{n}(m)\right) \in\left(X_{1} \oplus \cdots \oplus X_{m}\right)_{Z}$ with $\left(x_{n}\right)$ bounded, then

$$
A^{*}\left(x_{n}\right) \geq\left\|\left(A^{*}\left(x_{n}(1)\right), \ldots, A^{*}\left(x_{n}(m)\right)\right)\right\|_{Z} .
$$

If $\left(x_{n}\right)$ is finite the inequality is obvious. Suppose then that $\left(x_{n}\right)$ is an infinite sequence. It clearly suffices to show that

$$
\gamma\left(y_{n}\right) \geq\left\|\left(A^{*}\left(x_{n}(1)\right), \ldots, A^{*}\left(x_{n}(m)\right)\right)\right\|_{Z}
$$

for any infinite subsequence $\left(y_{n}\right)$ of $\left(x_{n}\right)$. Supposing we had such a subsequence, for any $r<1$ lemma 2.3 will enable us to obtain a further subsequence $\left(z_{n}\right)$ so that $\operatorname{sep}\left(z_{n}(i)\right) \geq r A^{*}\left(x_{n}(i)\right)$ for any $i$. This readily gives the above inequality.

Proposition 4.3. Suppose that $X_{1}, \ldots, X_{m}$ all have SSuP. Then so does $X=$ $\left(X_{1} \oplus \cdots \oplus X_{m}\right)_{Z}$.

Proof. Suppose $x_{n}=\left(x_{n}(1), \ldots, x_{n}(m)\right) \in X, x_{n} \stackrel{w}{\longrightarrow} 0$ and liminf $\left\|x_{n}\right\|>0$. Let $h_{i}=\limsup _{n}\left\|x_{n}(i)\right\|$. In establishing the SSuP condition it can be checked that those coordinates for which $h_{i}=0$ can be disregarded. Consider this done.

For $0 \leq l<h_{i}$ put $N_{l}^{i}=\left\{n \in \mathbb{N}:\left\|x_{n}(i)\right\| \geq l\right\}$ and

$$
\begin{aligned}
r_{i}(l)=\inf \{r: & \limsup \left\|y_{n}(i)\right\| \leq r \operatorname{diam}\left(y_{n}(i)\right) \\
& \text { for all subsequences } \left.\left(y_{n}(i)\right) \text { of }\left(x_{n}(i)\right)_{n \in N_{l}^{i}}\right\} .
\end{aligned}
$$

Note that the above infimum is achieved and that $r_{i}(l)$ is decreasing on $\left[0, h_{i}\right)$. For convenience put $r_{i}\left(h_{i}\right)=\lim _{l \rightarrow h_{i}^{-}} r_{i}(l)$. Then $r_{i}(l)$ is decreasing on $\left[0, h_{i}\right]$, and since $\left(x_{n}\right)$ is bounded it is easily seen that $r_{i}\left(h_{i}\right)>0$. Also, since the $X_{i}$ have SSuP, $r_{i}(l)<1$ for $l \in\left(0, h_{i}\right)$.

Choose $\lambda \in(0,1)$. We begin by showing that there exists $r \in(0,1)$ so that if $\left\|\left(l_{1}, \ldots, l_{m}\right)\right\| \geq \lambda \liminf \left\|x_{n}\right\|$ and $0 \leq l_{i} \leq h_{i}$, then

$$
\left\|\left(\frac{l_{1}}{r_{1}\left(l_{1}\right)}, \ldots, \frac{l_{m}}{r_{m}\left(l_{m}\right)}\right)\right\| \geq \frac{1}{r}\left\|\left(l_{1}, \ldots, l_{m}\right)\right\| .
$$

Indeed, to argue for a contradiction, suppose no such $r$ exists. Then for every $i \in\{1, \ldots, m\}$ there exists a sequence $\left(l_{i}^{n}\right)$ with $0 \leq l_{i}^{n} \leq h_{i}$ and $\left\|\left(l_{1}^{n}, \ldots, l_{m}^{n}\right)\right\| \geq$ $\lambda \liminf \left\|x_{n}\right\|$ so that

$$
\left\|\left(\frac{l_{1}^{n}}{r_{1}\left(l_{1}^{n}\right)}, \ldots, \frac{l_{m}^{n}}{r_{m}\left(l_{m}^{n}\right)}\right)\right\| \leq \frac{n+1}{n}\left\|\left(l_{1}^{n}, \ldots, l_{m}^{n}\right)\right\| .
$$

We can also assume that $l_{i}^{n} \rightarrow l_{i}$ and $r_{i}\left(l_{i}^{n}\right) \rightarrow r_{i}$. Clearly $r_{i}>0$ for all $i, 0 \leq l_{i} \leq h_{i}$ and $\left\|\left(l_{1}, \ldots, l_{m}\right)\right\| \geq \lambda \liminf \left\|x_{n}\right\|$. By continuity

$$
\left\|\left(\frac{l_{1}}{r_{1}}, \ldots, \frac{l_{m}}{r_{m}}\right)\right\| \leq\left\|\left(l_{1}, \ldots, l_{m}\right)\right\| .
$$

Now suppose $i$ satisfies $l_{i}>0$ (such an $i$ exists, since $\left.\left\|\left(l_{1}, \ldots, l_{m}\right)\right\|>0\right)$. Since $l_{i}^{n} \rightarrow$ $l_{i}>0$ and $r_{i}(l)$ is decreasing, for sufficiently large $n$ we have $r_{i}\left(l_{i}^{n}\right) \leq r_{i}\left(l_{i} / 2\right)<1$, the last inequality due to the SSuP of $X_{i}$. Thus $r_{i}<1$. Put $s=\max \left\{r_{i}: l_{i}>0\right\}<$ 1. Then

$$
\left\|\left(\frac{l_{1}}{r_{1}}, \ldots, \frac{l_{m}}{r_{m}}\right)\right\| \geq \frac{1}{s}\left\|\left(l_{1}, \ldots, l_{m}\right)\right\|>\left\|\left(l_{1}, \ldots, l_{m}\right)\right\|,
$$

contradicting the above. 
We now show that if $z_{n}=\left(z_{n}(1), \ldots, z_{n}(m)\right)$ is a subsequence of $\left(x_{n}\right)$, then

$$
\lim \sup \left\|z_{n}\right\| \leq r \operatorname{diam}\left(z_{n}\right),
$$

showing that $X$ has SSuP. We can assume that $\left\|z_{n}(i)\right\| \rightarrow l_{i}^{\prime}$ for all $i$. Then

$$
\left\|\left(l_{i}^{\prime}, \ldots, l_{m}^{\prime}\right)\right\|=\lim \left\|z_{n}\right\| \geq \liminf \left\|x_{n}\right\| .
$$

Suppose $\mu$ satisfies $\lambda<\mu<1$. Put $l_{i}=\mu l_{i}^{\prime}$. Then $\left\|\left(l_{1}, \ldots, l_{m}\right)\right\| \geq \lambda \liminf \left\|x_{n}\right\|$. We can then further assume that $\left\|z_{n}(i)\right\| \geq l_{i}$ (even if $l_{i}=0$, of course). Now $0 \leq l_{i}<h_{i}$, and from the definition of $r_{i}(l)$ we can assume that

Then

$$
A^{*}\left(z_{n}(i)\right) \geq \frac{l_{i}^{\prime}}{r_{i}\left(l_{i}\right)} \geq \frac{l_{i}}{r_{i}\left(l_{i}\right)}
$$

$$
\begin{aligned}
A^{*}\left(z_{n}\right) & \geq \|\left(A^{*}\left(z_{n}(1)\right), \ldots, A^{*}\left(z_{n}(m)\right) \|\right. \\
& \geq\left\|\left(\frac{l_{1}}{r_{1}\left(l_{1}\right)}, \ldots, \frac{l_{m}}{r_{m}\left(l_{m}\right)}\right)\right\| \\
& \geq \frac{1}{r}\left\|\left(l_{1}, \ldots, l_{m}\right)\right\| \\
& =\frac{\mu}{r}\left\|\left(l_{1}^{\prime}, \ldots, l_{m}^{\prime}\right)\right\| \\
& =\frac{\mu}{r} \lim \left\|z_{n}\right\| .
\end{aligned}
$$

Since $\mu$ was arbitrary we have $A^{*}\left(z_{n}\right) \geq(1 / r) \lim \left\|z_{n}\right\|$, and the proof is complete.

We note here that in [5] it is shown that if $X_{1}, \ldots, X_{n}$ are Banach spaces then

$$
W C S\left(\left(X_{1} \oplus X_{2} \cdots \oplus X_{n}\right)_{Z}\right)=\min \left\{W C S\left(X_{i}\right): 1 \leq i \leq n\right\} .
$$

We now consider infinite product results. We restrict ourselves to considering substitution spaces with bases. Suppose $X$ is a Banach space with a 1-unconditional Schauder basis $\left\{e_{n}\right\}$. That is, if $\sum_{n=1}^{\infty} b_{n} e_{n} \in X$ then, if $\left|a_{n}\right| \leq\left|b_{n}\right|$ implies that $\sum_{n=1}^{\infty} a_{n} e_{n}$ exists and $\left\|\sum_{n=1}^{\infty} a_{n} e_{n}\right\| \leq\left\|\sum_{n=1}^{\infty} b_{n} e_{n}\right\|$. In particular, $X$ is a Banach lattice with the coordinatewise ordering.

If $\left(X_{n}\right)$ is a sequence of Banach spaces, we denote by $\bigoplus_{X} X_{n}$ the space $\left\{\left(x_{n}\right)\right.$ : $x_{n} \in X_{n}, \sum_{n=1}^{\infty}\left\|x_{n}\right\| e_{n}$ exists $\}$ with norm $\left\|\left(x_{n}\right)\right\|=\left\|\sum_{n=1}^{\infty}\right\| x_{n}\left\|e_{n}\right\|$. If $M$ is an Orlicz function and $h_{M}$ the associated Orlicz sequence space, we denote the $h_{M}$ product of the spaces $X_{n}$ by $\bigoplus_{M} X_{n}$. If $M=x^{p}$ with $1 \leq p<\infty$, we use $\bigoplus_{\ell_{p}} X_{n}$ to denote the product space.

Proposition 4.4. Suppose that $X_{n}$ has $S S u P$ for all $n$. Then so does $X=\bigoplus_{\ell_{1}} X_{n}$.

Proof. Suppose that $\left(x_{n}\right)$ is a sequence in $X$ with $\liminf \left\|x_{n}\right\|>0, x_{n} \stackrel{w}{\longrightarrow} 0$. We will denote by $P_{n}$ the projection onto the subspace of $X$ naturally identified with $X_{n}$, and put $S_{n}=\sum_{i=1}^{n} P_{i}$.

We can clearly assume that there exists $\delta>0$ so that $\left\|x_{n}\right\| \geq \delta$ for all $n$. We now claim that there exist $m \in \mathbb{N}$ and $\epsilon>0$ so that $\left\|S_{m}\left(x_{n}\right)\right\| \geq \epsilon$ for all $n$. Indeed, suppose not. Then there would exist a subsequence $\left(y_{n}\right)$ of $\left(x_{n}\right)$ and a sequence $\left(z_{n}\right)$ that is supported on disjoint $\ell_{1}$ blocks satisfying $\left\|z_{n}-y_{n}\right\| \rightarrow 0$. That is, there exist disjoint intervals $I_{n}$ of natural numbers so that $\left(\sum_{q \in I_{n}} P_{q}\right)\left(z_{n}\right)=z_{n}$. Note that $z_{n} \stackrel{w}{\longrightarrow} 0$ and liminf $\left\|z_{n}\right\|>0$. We can obviously assume that $\left\|z_{n}\right\|>0$ for all 
$n$. If we now put $w_{n}=\left\|z_{n}\right\| e_{n}$, where $e_{n}$ is the $n^{\text {th }}$ element of the usual unit basis of $\ell_{1}$, then $\left(z_{n}\right)$ is equivalent to $\left(w_{n}\right)$. Indeed, the correspondence $z_{n} \leftrightarrow w_{n}$ extends to a linear isometry of $\overline{\operatorname{span}}\left(z_{n}\right)$ with $\overline{\operatorname{span}}\left(w_{n}\right)$. But since $\lim \inf \left\|w_{n}\right\|>0,\left(w_{n}\right)$ cannot be a weak null sequence, a contradiction.

By proposition 4.3, $\left(X_{1} \oplus \cdots \oplus X_{m}\right)_{\ell_{1}}$ has SSuP. Thus there exists $r<1$ so that $\lim \sup \left\|y_{n}\right\| \leq r \operatorname{diam}\left(y_{n}\right)$ for any subsequence $\left(y_{n}\right)$ of $\left(S_{m}\left(x_{n}\right)\right)$. Now suppose that $\left(z_{n}\right)$ is a subsequence of $\left(x_{n}\right), z_{n}=S_{m}\left(z_{n}\right)+\left(I-S_{m}\right)\left(z_{n}\right)$. Suppose that $\left\|S_{m}\left(z_{n}\right)\right\| \rightarrow a$ and $\left\|\left(I-S_{m}\right)\left(z_{n}\right)\right\| \rightarrow b$.

Then

$$
\begin{aligned}
A^{*}\left(z_{n}\right) & \geq A^{*}\left(S_{m}\left(z_{n}\right)\right)+A^{*}\left(\left(I-S_{m}\right)\left(z_{n}\right)\right) \\
& \geq \frac{a}{r}+b \\
& =a+b+a\left(\frac{1}{r}-1\right) \\
& \geq a+b+\epsilon\left(\frac{1}{r}-1\right) \\
& =\lim \left\|z_{n}\right\|+\epsilon\left(\frac{1}{r}-1\right) .
\end{aligned}
$$

This gives SSuP for $X$ by 3.4.

Corollary 4.5. Bynum's condition and SSuP are distinct.

Proof. Put $X_{n}=\ell_{n+1}$ in the above proposition. The fact that $W C S\left(\ell_{n}\right)=2^{1 / n}$ if $1<n<\infty$ gives the result.

Below we give infinite product results for Bynum's condition and asymptotic (P). First we need to recall some further definitions. A Banach lattice $X$ is said to be monotone if, when $a, b \in X$ with $0 \leq a \leq b$ and $\|b-a\|>0$, then $\|b\|>\|a\| . X$ is called uniformly monotone if for each $\epsilon>0$ there exists $\delta>0$ so that if $a \geq 0$, $\|a\| \leq 1$ and $b \geq a$ with $\|b-a\| \geq \epsilon$, then $\|b\| \geq\|a\|+\delta$. In [1] it is shown that this condition is equivalent to the same condition with the inequality $\|a\| \leq 1$ replaced by equality.

The following proposition will be of interest later. If $X$ has a 1-unconditional basis $\left(e_{n}\right)$ and $A \subseteq \mathbb{N}$, we denote by $P_{A}$ the natural projection onto $\overline{\operatorname{span}}\left\{e_{n}\right\}_{n \in A}$. If $A$ is the empty set, we take $P_{A}$ to be the projection onto the trivial one-element subspace.

Proposition 4.6. Suppose that $X$ has a 1-unconditional Schauder basis, and consider it as a Banach lattice under the associated ordering. Then the following are equivalent.

(1) $X$ is uniformly monotone.

(2) If $\epsilon>0$, then there exists $\delta>0$ so that if $A \subseteq \mathbb{N}$ and $x \in X$ with

$$
\left\|P_{A} x\right\|=1 \quad \text { and } \quad\left\|P_{\mathbb{N} \backslash A} x\right\| \geq \epsilon
$$

then $\|x\| \geq 1+\delta$.

(3) If $\epsilon>0$, then there exits $\delta>0$ so that if $0 \leq a \leq b,\|b\| \leq 1$ and $\|a\| \geq \epsilon$ then $\|b-a\| \leq 1-\delta$.

(4) If $\epsilon>0$, then there exists $\delta>0$ so that if $A \subseteq \mathbb{N}$ and $x \in X$ with 


$$
\left\|P_{A} x\right\| \geq \epsilon \quad \text { and } \quad\left\|P_{\mathbb{N} \backslash A} x\right\| \geq 1-\delta
$$

then $\|x\|>1$.

Proof. That (1) implies (2) is obvious.

$(2) \Rightarrow(4)$ : Suppose that (4) fails, so there exist a sequence $\left(x_{n}\right)$, subsets $A_{n}$ of $\mathbb{N}$, and $\epsilon>0$ so that $\left\|P_{A_{n}} x_{n}\right\| \geq \epsilon,\left\|P_{\mathbb{N} \backslash A_{n}} x_{n}\right\| \rightarrow 1$ and $\left\|x_{n}\right\| \leq 1$. Then put

$$
y_{n}=\frac{x_{n}}{\left\|P_{\mathbb{N} \backslash A_{n}} x_{n}\right\|},
$$

so that $\liminf \left\|P_{A_{n}} y_{n}\right\| \geq \epsilon,\left\|P_{\mathbb{N} \backslash A_{n}} y_{n}\right\|=1$ and $\lim \sup \left\|y_{n}\right\| \leq 1$. But this will contradict (2).

$(4) \Rightarrow(3)$ : The proof is a specilization of one used in [13] to obtain a more general result in Banach lattice function spaces. Suppose that (3) fails. Then there are sequences $\left(x_{n}\right)$ and $\left(y_{n}\right)$ in $X$ so that $x_{n} \in B_{X}, 0 \leq y_{n} \leq x_{n},\left\|y_{n}-x_{n}\right\| \rightarrow 1$ and $\left\|y_{n}\right\| \geq \epsilon$.

Choose a sequence $\left(\epsilon_{n}\right)$ from $(0, \epsilon)$ so that $\epsilon_{n} \rightarrow 0$ and

$$
\frac{1-\left\|x_{n}-y_{n}\right\|}{\epsilon_{n}} \rightarrow 0 \text {. }
$$

Put

$$
C_{n}:=\left\{m \in \mathbb{N}: P_{m}\left(y_{n}\right) \geq \epsilon_{n} P_{m}\left(x_{n}\right)\right\}
$$

Now

$$
\left\|P_{\mathbb{N} \backslash C_{n}}\left(y_{n}\right)\right\| \leq \epsilon_{n}\left\|P_{\mathbb{N} \backslash C_{n}}\left(x_{n}\right)\right\| \leq \epsilon_{n} .
$$

Thus

$$
\left\|P_{C_{n}}\left(x_{n}\right)\right\| \geq\left\|P_{C_{n}}\left(y_{n}\right)\right\| \geq \epsilon-\epsilon_{n} \rightarrow \epsilon
$$

But,

$$
\begin{aligned}
\left\|x_{n}-y_{n}\right\| & \leq\left\|x_{n}-P_{C_{n}}\left(y_{n}\right)\right\| \\
& \leq\left\|P_{\mathbb{N} \backslash C_{n}}\left(x_{n}\right)+\left(1-\epsilon_{n}\right) P_{C_{n}}\left(x_{n}\right)\right\| \\
& =\left\|\epsilon_{n} P_{\mathbb{N} \backslash C_{n}}\left(x_{n}\right)+\left(1-\epsilon_{n}\right) x_{n}\right\| \\
& \leq \epsilon_{n}\left\|P_{\mathbb{N} \backslash C_{n}}\left(x_{n}\right)\right\|+1-\epsilon_{n} .
\end{aligned}
$$

Thus,

$$
\left\|P_{\mathbb{N} \backslash C_{n}}\left(x_{n}\right)\right\| \geq 1-\frac{1-\left\|x_{n}-y_{n}\right\|}{\epsilon_{n}} \rightarrow 1,
$$

which contradicts (4).

$(3) \Rightarrow(1)$ : Suppose that (1) fails, so that we obtain $\epsilon>0$ and sequences $\left(a_{n}\right),\left(b_{n}\right)$ so that $\left\|a_{n}\right\| \leq 1,0 \leq a_{n} \leq b_{n},\left\|b_{n}-a_{n}\right\| \geq \epsilon$ but $\left\|b_{n}\right\|-\left\|a_{n}\right\| \rightarrow 0$. Without loss of generality we can asssume that $\left\|b_{n}\right\| \rightarrow r$ and $\left\|a_{n}\right\| \rightarrow r$. Note that $r>\epsilon / 2$. Put $b_{n}^{\prime}=b_{n} /\left\|b_{n}\right\|$ and $a_{n}^{\prime}=a_{n} /\left\|b_{n}\right\|$, so $0 \leq a_{n}^{\prime} \leq b_{n}^{\prime}$ and $\left\|b_{n}^{\prime}\right\|=1$. Now choose $\delta>0$ so that $\epsilon / r-\delta>0$. Put $c_{n}=b_{n}^{\prime}-a_{n}^{\prime}$. Then for sufficiently large $n,\left\|c_{n}\right\|>\epsilon / r-\delta$. But $\left\|a_{n}^{\prime}\right\| \rightarrow 1$, which together with the above will contradict (3).

Proposition 4.7. Suppose $X$ has a 1-unconditional Schauder basis $\left(e_{n}\right)$ with respect to which it is uniformly monotone. Then, if $\inf \left\{W C S\left(X_{n}\right): n \in \mathbb{N}\right\}>1$, $\operatorname{WCS}\left(\oplus_{X} X_{n}\right)>1$. 
Proof. Put $w:=\inf \left\{W C S\left(X_{n}\right): n \in \mathbb{N}\right\}$ and $Y:=\bigoplus_{X} X_{n}$. The uniform monotonicity of $X$ gives a $\delta>0$ so that if $a, b \in X, 0 \leq a \leq b,\|b\| \leq 3$ and $\|b-a\| \geq \max \{(1 / 4)(w-1), 1 / 4\}$, then $\|b\| \geq\|a\|+\delta$. Suppose now that $x_{n} \in Y$, $x_{n} \stackrel{w}{\longrightarrow} 0$ and $\left\|x_{n}\right\|=1$. We show that $\operatorname{diam}\left(x_{n}\right) \geq 1+\delta$.

We now use a technique of Benavides [5].

By 2.5 we can assume that $\lim _{n, m \rightarrow \infty(n \neq m)}\left\|x_{n}(i)-x_{m}(i)\right\|=l(i)$ exists for all $i$ (where $\left.x_{n}=\sum x_{n}(i) e_{i}\right)$. We can further assume that $\lim \left\|x_{n}(i)\right\|=a(i)$. Note that $\left\|\sum_{i=1}^{n} a(i) e_{i}\right\| \leq 1$ for all $n$, since $\left\|x_{n}\right\|=1$ for all $n$. Since $X$ is uniformly monotone with respect to the coordinatewise ordering given by the basis, it follows fairly readily that the basis is boundedly complete, and so $\sum a(i) e_{i}$ exists.

Now suppose that $1 / 8>\epsilon>0$. Then there exists $i_{1} \in \mathbb{N}$ so that $\left\|\sum_{i>i_{1}} a(i) e_{i}\right\|<$ $\epsilon$. Choose $n_{1} \in \mathbb{N}$ large enough so that, for any $m>n_{1}$,

$$
\|\| \sum_{i \leq i_{1}}\left\|x_{m}(i)\right\| e_{i}\|-\| \sum_{i \leq i_{1}} a(i) e_{i} \| \mid<\epsilon,
$$

with

$$
\left\|\left|\left\|\sum_{i \leq i_{1}}\right\| x_{n_{1}}(i)-x_{m}(i)\left\|e_{i}\right\|-\left\|\sum_{i \leq i_{1}} l(i) e_{i}\right\|\right|<\epsilon .\right.
$$

There exists $i_{2} \in \mathbb{N}$ so that $\left\|\sum_{i>i_{2}}\right\| x_{n_{1}}(i)\left\|e_{i}\right\|<\epsilon$. Choose $n_{2}>n_{1}$ large enough so that $\left\|\sum_{1=i_{1}+1}^{i_{2}}\right\| x_{n_{2}}(i)\left\|e_{i}\right\|<\epsilon$ (using the fact that $\left\|\sum_{i>i_{1}} a(i) e_{i}\right\|<\epsilon$ ). Now

$$
\left\|x_{n_{1}}-x_{n_{2}}\right\|>\left\|\sum_{i \leq i_{1}} l(i) e_{i}+\sum_{i=i_{1}+1}^{i_{2}}\right\| x_{n_{1}}(i)\left\|e_{i}+\sum_{i>i_{2}}\right\| x_{n_{2}}(i)\left\|e_{i}\right\|-3 \epsilon
$$

and

$$
\begin{gathered}
\left\|\sum_{i \leq i_{1}} a(i) e_{i}+\sum_{i=i_{1}+1}^{i_{2}}\right\| x_{n_{1}}(i)\left\|e_{i}\right\|>1-2 \epsilon, \\
\left\|\sum_{i \leq i_{1}} a(i) e_{i}+\sum_{i=i_{1}+1}^{i_{2}}\right\| x_{n_{2}}(i)\left\|e_{i}\right\|>1-2 \epsilon .
\end{gathered}
$$

Case 1. $\left\|\sum a(i) e_{i}\right\| \geq 1 / 2$. Setting

$$
a:=\sum_{i \leq i_{1}} l(i) e_{i}+\sum_{i=i_{1}+1}^{i_{2}}\left\|x_{n_{1}}(i)\right\| e_{i}+\sum_{i>i_{2}}\left\|x_{n_{2}}(i)\right\| e_{i}
$$

and

$$
b:=\sum_{i \leq i_{1}} a(i) e_{i}+\sum_{i=i_{1}+1}^{i_{2}}\left\|x_{n_{1}}(i)\right\| e_{i}
$$


$0 \leq a \leq b,\|b\| \leq 3$, we get

$$
\begin{aligned}
\|a-b\| & \geq(w-1)\left\|\sum_{i \leq i_{1}} a(i) e_{i}\right\| \\
& \geq(w-1)(1 / 2-\epsilon) \\
& \geq(w-1) 1 / 4
\end{aligned}
$$

Thus $\|b\| \geq\|a\|+\delta \geq 1-2 \epsilon+\delta$ and $\left\|x_{n_{1}}-x_{n_{2}}\right\| \geq 1-5 \epsilon+\delta$. Since $\epsilon$ was arbitary, this gives the result.

Case 2. $\left\|\sum a(i) e_{i}\right\|<1 / 2$. Then $\left\|\sum_{i>i_{2}}\right\| x_{n_{2}}(i)\left\|e_{i}\right\|>1-2 \epsilon-1 / 2=1 / 2-2 \epsilon$. Now, for $a$ and $b$ as above, $\|a-b\| \geq\left\|\sum_{i>i_{2}}\right\| x_{n_{2}}(i)\left\|e_{i}\right\|>1 / 2-2 \epsilon>1 / 4$, so $\|b\|>\|a\|+\delta$, and we proceed as in case 1 .

Proposition 4.8. If $X$ is as in the above proposition and $X_{n}$ has asymptotic $(P)$ for each $n \in \mathbb{N}$, then so does $\bigoplus_{X} X_{n}$.

Proof. Suppose that $x_{n} \in X,\left\|x_{n}\right\|=1$ and $x_{n} \stackrel{w}{\longrightarrow} 0$. For a contradiction suppose that $\gamma\left(x_{n}\right)=1$ (noting that $\gamma\left(x_{n}\right) \geq 1$ for any such sequence $\left(x_{n}\right)$ by weak lower semicontinuity). As in the proof of the above theorem, we can further assume that $\lim \left\|x_{n}(i)\right\|=a(i)$ and $\lim _{n, m \rightarrow \infty(n \neq m)}\left\|x_{n}(i)-x_{m}(i)\right\|=l(i)$ exist. The assumption on $\left(x_{n}\right)$ implies that $\left\|\sum_{i=1}^{n} l(i) e_{i}\right\| \leq 1$ for all $n$. Note also that $\left\|\sum a(i) e_{i}\right\| \leq 1$. The proof now divides into two cases.

Case 1. $\left\|\sum a(i) e_{i}\right\|=1$. Obviously there exists $i \in \mathbb{N}$ so that $a(i)>0$. Thus, since $X_{i}$ has asymptotic $(\mathrm{P}), l(i)>a(i)$. Monotonicity of the norm now produces a contradiction.

Case 2. $\alpha:=\left\|\sum a(i) e_{i}\right\|<1$. We argue in a similar way to the proof of the above theorem, replacing $1 / 2$ by $\alpha$ to obtain $\gamma\left(x_{n}\right) \geq 1+\delta$ for some $\delta>0$, a contradiction.

Example 4.9. If $M$ is a nondegenerate Orlicz function satisfying the $\triangle_{2}$ condition at 0 , then it is shown in [9] that it has the Gossez-Lami Dozo (GLD) property. That is, for every $\epsilon>0$ there exists a $\delta>0$ so that if $x \in X_{M}$ with $\left\|\sum_{n=1}^{m} P_{n} x\right\|=1$ and $\left\|\left(I-\sum_{n=1}^{m} P_{n}\right) x\right\| \geq \epsilon$, then $\|x\| \geq 1+\delta$. From the definition of the norm in $X_{M}$ it is not difficult to see that this property is equivalent to condition (2) of 4.6 for $X_{M}$. Thus 4.6 gives that $X_{M}$ is uniformly monotone, and so $X_{M}$ suffices for $X$ in the previous two propositions. We note that in [5] the result of 4.7 was achieved when the substitution space is an Orlicz space as above, and better estimates on the constants were achieved. In particular, the arguments return the best estimate for $W C S\left(\bigoplus_{X_{M}} X_{n}\right)$ in terms of the $W C S\left(X_{n}\right)$ when the Orlicz function is of the form $x^{p}$ for $1 \leq p<\infty$. We also note that in [9] it was proved, with the substitution space as above, that if the $X_{n}$ have UKK then the product has weak normal structure. As noted in section 3 , the $X_{n}$ have asymptotic $(\mathrm{P})$, and so 4.8 extends the result from [9].

Example 4.10. If $X$ is a uniformly convex space with a 1-unconditional basis, then it is well known that $X$ is uniformly monotone. Thus 4.7 generalizes a result fom $[5]$.

The proof of the following corollary uses the same example that served in [15] to separate asymptotic (P) from Bynum's condition.

Corollary 4.11. Asymptotic $(P)$ and SuP are distinct properties. 
Proof. Put $X_{n}=\ell_{n+1}$ and $X=\ell_{2}$ in the above proposition. The resulting $Y:=$ $\bigoplus_{\ell_{2}} X_{n}$ then has asymptotic $(\mathrm{P})$. That it fails to have SuP can be verified directly. However, $W C S\left(\ell_{p}\right)=2^{1 / p} \rightarrow 1$ gives $W C S(Y)=1$, which combined with the fact that $Y$ is reflexive, thus Asplund, allows us to deduce the required fact from theorem 3.5 .

We have not achieved any infinite product results for property $(\mathrm{P})$, but we ask whether $(\mathrm{P})$ is preserved under infinite $\ell_{p}$ products for $1 \leq p<\infty$. We also reiterate the question from [5] about the exact relationship between $\inf W C S\left(X_{n}\right)$, $W C S(X)$ and $W C S\left(\bigoplus_{X} X_{n}\right)$, for a substitution space $X$.

\section{REFERENCES}

1. M. A. Akcoglu and L. Sucheston, On uniform monotonicity of norms and ergodic theorems in function spaces, Proc. Conf. Commemorating First Centennial Circ. Mat. Palermo, Rend. Circ. Mat. Palermo (2) Suppl. No. 8 (1985), 325-335. MR 88m:46029

2. J. Barwise (editor), Handbook of mathematical logic, Studies in Logic and the Foundations of Mathematics 90, North-Holland, Amsterdam, 1977. MR 56:15351

3. L. P. Belluce, W. A. Kirk and E. F. Steiner, Normal structure in Banach spaces, Pacific J. Math. 26 (1968), 433-440. MR 38:1501

4. T. Dominguez Benavides, Some properties of the set and ball measures of non-compactness and applications, J. London Math. Soc. 34 (1986), 120-128. MR 87k:47124

5. T. Dominguez Benavides, Weak uniform normal structure in direct sum spaces, Studia Math. 103 (1992), 283-290. MR 94c:46024

6. W. L. Bynum, Normal structure coefficients for Banach spaces, Pacific J. Math. 86 (1980), 427-436. MR 81m:46030

7. D. van Dulst, Reflexive and superreflexive Banach spaces, Mathematical Centre Tracts, Vol. 102, Mathematische Centrum, Amsterdam, 1978. MR 80d:46019

8. D. van Dulst and B. Sims, Fixed points of nonexpansive mappings and Chebyshev centres in Banach spaces with norms of type $K K$, Banach space theory and its applications, Springer Verlag Lecture Notes in Maths., 991, (1983), 35-43. MR 84i:46027

9. D. van Dulst and V. de Valk, $(K K)$-properties, normal structure and fixed points of nonexpansive mappings in Orlicz sequence spaces, Can. J. Math 28 (1986), 728-750. MR 87i:46049

10. K. Goebel and W. A. Kirk, Topics in metric fixed point theory, Cambridge University Press, 1990. MR 92c: 47070

11. R. Huff, Banach spaces which are nearly uniformly convex, Rocky Mountain Journal of Math. 10 (1980), 743-749. MR 82b:46016

12. A. Jimenez-Melado, Stability of weak normal structure in James quasi reflexive space, Bull. Austral. Math. Soc. 46 (1992), 367-372. MR 93m:46011

13. D. Kutzarova and T. Landes, Nearly uniform convexity of infinite direct sums, Boll. Un. Mat. Ital. (A) (7) 9 (1995), 527-534. MR 97c:46012

14. D. Kutzarova, E. Maluta, and S. Prus, Property $(\beta)$ implies normal structure of the dual space, Rend. Circ. Mat. Palermo (2) 41 (1992), 353-368. MR 94i:46020

15. T. Landes, Permanence properties of normal structure, Pacific J. Math. 110 (1984), 125-143. MR 86e: 46014

16. T. Landes, Normal structure and the sum-property, Pacific J. Math. 123 (1986), 127-147. MR 87h:46043

17. Z. Opial, Weak convergence of the sequence of successive approximations for nonexpansive mappings, Bull. A. M. S. 73 (1967), 591-597. MR 35:2183

18. S. Prus, On Bynum's fixed point theorem, Atti Sem. Mat. Fis. Univ. Modena 38 (1990), 535-545. MR 91k:47146

19. B. Sims and M. Smyth, On non-uniform conditions giving weak normal structure, Quaestiones Mathematicae 18 (1995), 9-19. MR 96e:46021

20. K. Tan and $\mathrm{H}$. Xu, On fixed point theorems of nonexpansive mappings in product spaces, Proc. A. M. S. 113 (1991), 983-989. MR 92e:47074

21. D. Tingley, The normal structure of James quasireflexive space, Bull. Austral. Math. Soc. 42 (1990), 95-100. MR 91h:46038 
22. G. Zhang, Weakly convergent sequence coefficient of product space, Proc. A. M. S. 117 (1993), 637-643. MR 93d:46037

23. W. Zhao, Remarks on various measures of noncompactness, J. Math. Anal. Appl. 174 (1993), 290-297. MR 94b:54007

Department of Mathematics, University of Newcastle, Newcastle, NSW 2308, AusTRALIA

E-mail address: bsims@maths.newcastle.edu.au 